\title{
Rassf 3 is responsible in part for resistance to mammary tumor development in neu transgenic mice
}

\author{
ISABELLE C. JACQUEMART, ALISON E.B. SPRINGS and WEN Y.CHEN \\ Department of Biological Sciences, Clemson University, Clemson, SC 29634; \\ Oncology Research Institute, Greenville Hospital System, Greenville, SC 29605, USA
}

Received August 20, 2008; Accepted October 21, 2008

DOI: 10.3892/ijo_00000177

\begin{abstract}
MMTV/neu transgenic mouse line is a welldocumented model for studying HER2/neu-related breast cancer. Approximately $80 \%$ of these mice develop mammary tumors by 11 months of age, whereas a small percentage appears to have naturally acquired resistance to HER2/neu tumorigenesis. To identify factors responsible for tumor resistance in these transgenic mice, comparative genetic profiling was used to screen alterations in gene expression in the mammary gland. A novel gene, the RAS association domain (RalGDS/AF-6) family 3 (Rassf3), which belongs to a family of RAS effectors and tumor suppressor genes, was identified. Data indicated 1) that Rassf3 is overexpressed in mammary gland of tumor-resistant MMTV/neu mice compared to tumor-susceptible MMTV/neu littermates or non-transgenic mice, and 2) Rassf3 is significantly up-regulated in neu-specific mouse mammary tumors compared to adjacent normal tissues. In vitro overexpression of RASSF3 inhibited cell proliferation in HER2/neu positive human and mouse breast cancer cell lines, possibly through induction of apoptosis. A novel MMTV/Rassf3-neu bi-transgenic mouse line, overexpressing Rassf3 and neu genes in mammary glands, was established. Mammary tumor incidence in bi-transgenic mice was delayed compared to their MMTV/ne $u^{+/-}$littermates. These data suggest that Rassf3 may influence mammary tumor incidence in MMTV/neu transgenic mice.
\end{abstract}

Correspondence to: Dr Wen Y. Chen, Oncology Research Institute, Greenville Hospital System, 900 West Faris Road, Greenville, SC 29605, USA

E-mail:wchen@ghs.org

Abbreviations: MG, mammary gland; T, tumor; MMTV, mouse mammary tumor virus; HER2, human epidermal growth factor receptor 2; RASSF, Ras association domain (RalGDS/AF-6) family; R-MG, mammary gland from tumor-resistant MMTV/neu transgenic mice; S-NMG, normal mammary gland from tumorsusceptible MMTV/neu transgenic mice

Key words: HER2/neu mammary tumorigenesis, MMTV/neu transgenic mice, tumor suppressor gene, Rassf3

\section{Introduction}

HER2/neu is overexpressed in $20-30 \%$ of primary human breast cancers $(1,2)$ and is associated with a poor clinical outcome. The HER2/neu proto-oncogene encodes a transmembrane glycoprotein, termed ErbB2, which belongs to the human epidermal growth factor receptor family. Receptor activation and consequent signal transduction involves hetero- or homo-dimerization utilizing various ErbB receptor combinations. ErbB2 has no known ligand but forms active receptor complexes with co-receptors of the ErbB family. Excess ErbB2 receptors on the cell surface can lead to spontaneous formation of activated ErbB2 homodimers. The transforming potential of ErbB2 is closely related to its intrinsic tyrosine kinase activity (3). Upon dimerization, the protein kinase of each ErbB receptor monomer transphosphorylates a distinct set of tyrosine residues in the intracellular domain of its dimer partner (4) and the resulting phosphotyrosines serve as docking sites for proteins that contain conserved Src homology (SH) 2 domain or phosphotyrosinebinding domain (PTB). Two major downstream signaling pathways activated by the ErbB family are the RAS-RAFMAPK pathway and the PI3K-AKT survival pathway (5), which lead to activation of transcription factors that mediate cellular responses.

There is considerable evidence that aberrant RAS activation and signaling may promote breast cancer development $(6,7)$. RAS can be activated by persistent upstream signaling from EGFR, ErbB2 or estrogen receptors which are often overexpressed in breast cancer (7). RAS proteins belong to a large superfamily of small GTPases which are signal transduction proteins $(20-25 \mathrm{kDa})$. The RAS family includes the most common H-RAS, K-RAS, and N-RAS proteins which can interact with a wide array of effectors through the $R A S$ binding domain (RBD) to stimulate diverse cytoplasmic signaling pathways (8). Although RAS proteins are usually associated with loss of growth control and tumorigenic transformation (9), increasing evidence has demonstrated that RAS proteins have the ability to activate a variety of growth-inhibiting pathways including apoptosis and cell cycle arrest (10-13). These contrasting activities suggest that activation of oncogenes such as RAS can promote conflicting biological processes depending on the interaction with and activation of distinct effectors, cell type, context, signal nature and intensity and tissue origin (14). Four members of the Ras 
association domain (RalGDS/AF-6) family, RASSF1, RASSF2, RASSF4 (AD037) and NORE1 (or RASSF5) have been identified as RAS effectors and tumor suppressors which may be involved in the pathways mediating RAS growth-inhibitory effect (15-18).

Overexpression of unactivated neu in the mammary epithelium results in appearance of focal mammary adenocarcinomas with a latency of 120-337 days that metastasize with high frequency to the lung. For line N\#202, 50\% of female virgin mice develop mammary tumors by 7 months ( $\mathrm{t}_{50}=205$ days) and $\sim 80 \%$ by 11 months or older (3). The latency in tumor development in the MMTV/neu transgenic mice reflects the multistep nature of tumorigenesis and implies that additional genetic events beyond neu overexpression are required for mammary tissue transformation leading to tumor formation (3). To date, the majority of studies utilizing MMTV/neu transgenic mice have focused on the mechanism by which overexpression of HER2/neu oncogene in epithelial cells initiates tumorigenesis in the $80 \%$ of mice which develop tumors prior to 11 months of age.

In the present study, we used MMTV/neu virgin female transgenic mouse model but focused on the small proportion $(\sim 20 \%)$ of transgenic mice which do not develop mammary tumors by 11 months despite overexpression of the neu oncogene. These mice appear to have naturally acquired resistance to HER2/neu-tumorigenesis. This resistance to HER2/neu-tumorigenesis may not be complete resistance to tumor formation, but it is marked by a significant delay in tumor latency. We hypothesized that there may be one or more tumor suppressor genes that are activated or induced in the mammary glands of these tumor-resistant mice. Comparative microarray technology was used to identify gene expression alterations in the mammary gland of tumor-resistant $\mathrm{MMTV} /$ neu transgenic mice and to identify candidate genes which may be responsible for resistance to HER2/neu mammary tumor development. Among the genes differentially expressed, we identified a member of the RASSF family, mouse Rassf3, which shares $\sim 40 \%$ and $44 \%$ identity at the amino acid level with hRASSF1A and hRASSF1C protein isoforms, respectively. We found that Rassf3 is up-regulated in the mammary gland of 11-month-old tumor-resistant female MMTV/neu mice (R-MG) compared to tumor-susceptible mice (S-NMG) that bear tumors or age-matched non-transgenic mice (C). The Rassf3 gene was therefore selected as a candidate gene for further characterization of its role in HER2/neu breast cancer through in vitro and in vivo studies.

\section{Materials and methods}

Animal models. The mice used in this study were of FVB background. The MMTV/neu transgenic mouse line (N\#202) was purchased from The Jackson Laboratory (Bar Harbor, $\mathrm{ME)}$. This transgenic mouse line was developed by Guy et al (3) and is well characterized. Transgenic mice express inactivated rat neu oncogene under the transcriptional control of the MMTV promoter. The kinetics of tumor formation of this model reveal that $\sim 50 \%$ of virgin females develop mammary tumors by 205 days $\left(\mathrm{T}_{50}=205\right)$ and $\sim 80 \%$ by 337 days (3). A similar tumor rate was observed after expanding the N\#202 line in our animal facility. According to the experimental design, virgin female MMTV/neu transgenic mice were monitored for tumor development. Age-matched female littermates were divided into 2 groups: tumorsusceptible mice $(\mathrm{S})$ that were bearing mammary tumors and tumor-resistant mice $(\mathrm{R})$ that had no palpable tumor and microscopic-free of precursor lesions (Fig. 1A). A distinction was made between tumor tissue ( $\mathrm{T}$ ) and normal mammary gland (S-NMG) excised from a tumor-bearing mouse $(\mathrm{S})$. Non-transgenic FVB mice were used as control (C).

The MMTV/Rassf3 transgenic mouse line was established for this study. Mouse (723 bp) Rassf3-His Tag cDNA was subcloned into pMSG expression vector (pMSG/Rassf3-His Tag) and fused to MMTV-LTR promoter. The 3,180 bp MMTV-Rassf3-His Tag cDNA fragment was microinjected into fertilized mouse single-cell embryos of FVB mice (The Jackson Laboratory), following standard protocol (19). The tyrosinase gene, responsible for the agouti fur color was co-injected with the transgene of interest to have a color marker for transgene presence. MMTV/Rassf3 transgenic mice founder line \#13 was identified as transgene-positive and expanded. Animals were maintained in barrier facilities and cared for in accordance with the Institutional Animal Care and Use Committee (IACUC) guidelines and policies. Both male and female MMTV/Rassf3 transgenic mice presented no apparent overt phenotype up to currently 21 months of age. MMTV/Rassf3 heterozygotic male mice were cross-bred with MMTV/neu homozygous females to produce a MMTV/Rassf3-neu bi-transgenic line. All offspring were heterozygous for MMTV/neu ${ }^{+/-}$transgene and 50\% were positive for the MMTV/Rassf3 transgene and had the agouti fur color.

Transgene incorporation was confirmed by PCR analysis using genomic tail DNA and primers specific for the transgene. The forward primer (5'-TGT TTG TGT CTG TTC GCC AT-3') was designed to anneal to a region on the MMTV promoter sequence whereas the reverse primer (5'-TTT GCA GAG TTC CAT CTG CAC-3') was designed to anneal to a region located in the middle of the Rassf 3 sequence. Transgene incorporation was checked for every colored mouse from F1, F2 and F3 generations as well as for certain white littermate mice as negative control. Virgin female MMTV/Rassf3-neu bi-transgenic mice and virgin female MMTV/neu ${ }^{+-}$littermates were checked weekly for the development of mammary tumors. Kaplan-Meier survival curves were computed and statistical differences were calculated using the Log-rank test.

Affymetrix microarray. Mammary gland tissues were dissected from 11-month-old mice ( $\mathrm{n}=3$ per group) (S-NMG, R-MG and $\mathrm{C}$ ). For tumor-susceptible mice (S), typically, a piece of normal mammary gland was collected from the opposite site of the tumor-bearing gland and named 'S-NMG'. S-NMG samples were not histologically confirmed but selected by a careful visual examination under dissecting microscope to remove all potential lesions from the collected samples. Only a piece of the whole mammary gland was used for RNA or protein extractions. Total RNA was extracted and pooled per group to control for individual variations between mice. RNA samples were purified with Qiagen RNeasy cleanup procedure following the manufacturer's instructions (Qiagen, Valencia, CA). The purity and quantity of each sample was assessed on 
an Agilent Technologies bioanalyzer and the RNA 6000 nano lab chip, following the manufacturer's instructions (Agilent Technologies, Santa Clara, CA). Keck Affymetrix Resource Group at Yale University (http://info.med.yale.edu/wmkeck/ affymetrix) carried out sample processing (cDNA and cRNA preparation), array hybridization and array data analysis.

The GeneChip mouse expression MOE430A (Affymetrix) was employed for analysis and provided $\sim 22,000$ probe sets representing transcripts and variants from $>14,000$ wellcharacterized mouse genes. The Affymetrix microarray 5.0 (MAS) software was used to inspect hybridization artifacts and to detect changes in gene expression. Microsoft Excel and GeneSpring GX (Agilent Technologies) software programs were used for further data analysis. The microarray analysis was repeated with new total RNA samples prepared from a second set of mice in order to obtain two sets of data. Despite careful sample preparation, the two Affymetrix experiments conducted at different times through commercial source did not show significant overlap. There was a substantial overlapping in down-regulated genes as compared to that of up-regulated genes. We speculate that the large variation between the two microarray results may be due to the fact that some of the mammary gland tissues from tumor-resistant mice were in various pre-tumor stages microscopically due to the presence of neu oncogene. Nonetheless, based upon the literature review, we selected Rassf3 as the target for this study. Table I presents the first Affymetrix experiment, which indicated Rassf3 expression difference.

RNA isolation and RT-PCR analyses. Tissues were homogenized using a Polytron PT1200 motorized homogenizer (Polytron; Bad Wildbad, Germany) and RNA was isolated by TRIzol (Invitrogen, Carlsbad, CA) and chloroform extraction (Fisher Scientific, Pittsburgh, PA)/isopropyl alcohol precipitation (Sigma, St. Louis, MO). RNA was quantified by UV spectrophotometry and was reverse transcribed using the Reverse transcription-PCR kit (Promega, Madison, WI) with appropriate primers. RT-PCR analysis was carried in a Gene Amp PCR system 9700 (Applied Biosystems, Foster City, CA); conditions used were: $45 \mathrm{~min}$ at $48^{\circ} \mathrm{C}, 1 \mathrm{~min}$ at $95^{\circ} \mathrm{C}$, 35 cycles: $1 \mathrm{~min}$ at $94^{\circ} \mathrm{C}, 30 \mathrm{sec}$ at $54^{\circ} \mathrm{C}, 1 \mathrm{~min}$ at $72^{\circ} \mathrm{C}$, and a final extension step at $72^{\circ} \mathrm{C}$ for $6 \mathrm{~min}$. The PCR conditions for the Rassf3 gene were slightly different and consisted of 35 cycles: $1 \mathrm{~min}$ at $94^{\circ} \mathrm{C}, 1.5 \mathrm{~min}$ at $68^{\circ} \mathrm{C}$, and a final extension step at $72^{\circ} \mathrm{C}$ for $6 \mathrm{~min}$.

Specific pairs of primers were designed for each gene: mRassf3 (5'-GCTAGCATGAGCAGCGGCTACAGCAG-3' and 5'-ACCGGTGCCGGGCTTCCACACCTCGC-3'), $m$ Socs 2 (5'-TTGACTCATCTCCCATGACC-3' and 5'-GCTGCATT CGGAGATAGTCT-3'), mEtvl (5'-GGAGCAGAATGGA TGGATTTT-3' and 5'-GGGTTACTCATGTTAGTACAC-3') and G3PDH (5'-CCACAGTCCATGCCATCAC-3' and 5'-TCC ACCACCCTGTTGCTGTA-3'). RT-PCR products were resolved on $1 \%$ agarose gel. Amplification of the G3PDH housekeeping gene was performed simultaneously to demonstrate equal starting amounts of total RNA. RT-PCR analyses on total RNA samples from pairs of human tumors and adjacent normal tissues purchased from Ambion Inc. (Austin, TX), were conducted in duplicate with specific human RASSF3 primers (5'-CTAGCATGAGCAGCGGCTACAG
CAG-3' and 5'-GTCGACTTAATCAGGCTTCCACACCT-3'). The number of PCR cycles was limited to 25 . Gel electrophoresis photographs were analyzed with the Kodak 1D image analysis software (Eastman Kodak Company Molecular Imaging Systems; Rochester, NY) to compare the mean intensity of the bands. The mean intensity was set to $100 \%$ for the normal breast tissue. The data were calculated as relative percentage of RASSF 3 expression in the different samples and represent the mean \pm SEM of two independent experiments.

Cell lines. SKBR3, BT-474, MDA-MB-453, BT-483, MDAMB-134, T-47D, MCF-7, MDA-MB-468, MDA-MB-436 and MDA-MB-231 human breast cancer cell lines were purchased from the American Type Culture Collection (ATCC, Manassas, VA) and maintained as recommended by ATCC. HC11 murine mammary cell line was kindly provided by Dr Ameae Walker (University of California, Riverside, CA) and were maintained in RPMI Medium 1640 supplemented with $10 \%$ FBS, $1 \mathrm{mM}$ sodium pyruvate, $10 \mu \mathrm{g} / \mathrm{ml}$ insulin and $10 \mathrm{ng} / \mathrm{ml}$ EGF (Cambrex, East Rutherford, NJ). MCNeuA and N202Fb3 murine mammary cells, provided by Dr Mike Campbell (University of California, San Francisco, CA), were maintained in Dulbecco's modified Eagle medium (DMEM) supplemented with $10 \%$ FBS and $1 \mathrm{mM}$ sodium pyruvate.

Protein isolation and immunoblot analyses. Cell monolayers were washed with cold phosphate buffer saline (PBS) and lysed in lysis buffer $(50 \mathrm{mM}$ Tris $\cdot \mathrm{HCl}, \mathrm{pH} 7.4 ; 1 \%$ NP-40; $0.25 \%$ sodium deoxycholate; $150 \mathrm{mM} \mathrm{NaCl} ; 1 \mathrm{mM}$ EDTA, $\mathrm{pH} 8.0)$ containing protease inhibitors $(1 \mu \mathrm{g} / \mathrm{ml}$ aprotinin; $1 \mu \mathrm{g} / \mathrm{ml}$ leupeptin; $170 \mu \mathrm{g} / \mathrm{ml}$ PMSF; $180 \mu \mathrm{g} / \mathrm{ml}$ sodium orthovanadate) according to standard protocols. Protein content was measured using the Coomassie Plus Protein assay reagent (Pierce, Rockford, IL). Mammary gland, brain or mammary tumor tissues were excised, frozen on dry ice or immediately homogenized in lysis buffer $(50 \mathrm{mM}$ Tris $\cdot \mathrm{HCl}$, $\mathrm{pH} 7.4 ; 1 \%$ NP-40; $0.25 \%$ sodium deoxycholate; $150 \mathrm{mM}$ $\mathrm{NaCl} ; 1 \mathrm{mM}$ EDTA, pH 8.0) containing protease inhibitors (1 $\mu \mathrm{g} / \mathrm{ml}$ aprotinin; $1 \mu \mathrm{g} / \mathrm{ml}$ leupeptin; $1 \mu \mathrm{g} / \mathrm{ml}$ pepstatin; $170 \mu \mathrm{g} / \mathrm{ml}$ phenylmethylsulphonyl fluoride (PMSF); $180 \mu \mathrm{g} / \mathrm{ml}$ sodium orthovanadate; $50 \mathrm{mM}$ sodium fluoride), using a Polytron PT1200 motorized homogenizer. Lysates were processed as described above.

For immunoblotting, samples containing equal amounts of protein (50-100 $\mu \mathrm{g})$ were mixed with SDS-PAGE sample buffer and heated at $100^{\circ} \mathrm{C}$ for $5 \mathrm{~min}$, separated using $12 \%$ SDS-PAGE (Bio-Rad Laboratories, Hercules, CA) and transferred to a Hybond nitrocellulose (Amersham Biosciences, Piscataway, NJ) according to standard protocol. Membranes were blocked in tris-buffered saline (TBS) containing 5\% non-fat powdered milk and $0.05 \%$ Tween-20 for $1-2 \mathrm{~h}$ (TBS-T) before overnight incubation with the primary antibody in TBS-T/5\% milk at $4^{\circ} \mathrm{C}$ with gentle agitation. Membranes were washed with TBS-T for $5 \mathrm{~min}$, incubated with a secondary antibody in TBS-T/5\% milk for $2 \mathrm{~h}$ at room temperature and washed 3 times with TBS-T before incubation with the ECL ${ }^{\text {тм }}$ Western blotting detection reagents (Amersham Biosciences), as per the manufacturer's instructions. To visualize banding, membranes were exposed to Kodak 
Biomax MR film (Fisher Scientific) and developed with a Konica SRX-101A processor (Konica Minolta Medical Imagining, Wayne, NJ).

Primary antibodies were used at the following dilutions: 1:1000 anti-phospho-HER2/neu (Lab Vision; Fremont, CA) for cell lysate and 1:400 anti-phospho-HER2/neu (Santa Cruz Biotechnology, Santa Cruz, CA) for tissue homogenate; 1:1000 anti-HER2/neu (EMD Biosciences; Darmstadt, Germany); 1:1000 anti-RAS (Cell Signaling Technology); 1:10,000 antiß-actin (Millipore, Billerica, MA); 1:10,000 anti-ß-tubulin (Sigma). The secondary antibodies, goat anti-mouse IgG- and goat anti-rabbit IgG-horseradish peroxidase-conjugates were obtained from Bio-Rad Laboratories and used at 1:2000. A custom polyclonal antibody to mouse RASSF3 protein was produced by Proteintech Group Inc. (Chicago, IL) (http://www. PTGLab.com). Anti-RASSF3 antibody [RASSF3-Nt (S1228-1)] was produced from the following antigenic peptide sequence N'-MSSGYSSLEEDEDFFFTART-C and used at a dilution of 1:500 for immunoblot analyses. Control RASSF3 protein was produced in the lab using a previously published protocol (34) and used to test the specificity of the RASSF3-Nt antibody. The RASSF3 protein was immunoblotted as indicated above at different concentrations to determine the minimal amount of RASSF3 protein detected by this customized antibody.

Transient transfection, cell viability and apoptosis assay. SKBR3, BT-474, MCNeuA and HC11 cells were seeded to reach $60-80 \%$ confluency after $24 \mathrm{~h}$. Cells were starved for $2 \mathrm{~h}$ with Opti-MEM ${ }^{\circledR}$ reduced serum medium (Invitrogen) before transfection with 2 or $4 \mu \mathrm{g}$ of DNA plasmids [pcR3.1 vector, pcDNA3.1/Rassf3, pcDNA3.1/H-RAS (G12V) or pcDNA3.1/ H-RAS $(S 17 N)]$ using Lipofectamine 2000 Reagent (Invitrogen) according to the manufacturer's recommendations. Total amount of transfected DNA was maintained constant between compared samples. For cell viability counts, transfected cell lines were harvested $48 \mathrm{~h}$ after transfection, counted and re-seeded at equal concentration. After $24 \mathrm{~h}$, viability was assessed by MTS/PMS solution (Promega). Cell viability was calculated as a percentage of control, corresponding to pcR3.1 vector transfected cells. The data are represented as the mean \pm standard error of the mean (SEM) for all experiments. Transfection assays were performed in triplicate for BT-474, MCNeuA and HC11 cell lines and repeated 8 times for SKBR3 cell line.

For apoptosis assays, SKBR3 cells were seeded and transiently transfected with $2 \mu \mathrm{g}$ of pcR3.1 vector, $4 \mu \mathrm{g}$ of pcR3.1 vector, $2 \mu \mathrm{g}$ of pcDNA3.1/Rassf3 and $4 \mu \mathrm{g}$ of pcDNA3.1/Rassf3 as described above. Untransfected SKBR3 cells and camptothecin-treated SKBR3 cells $(50 \mu \mathrm{M} ; 24 \mathrm{~h})$ (Sigma) were used as negative control and positive control for induction of apoptosis, respectively. Each sample was prepared in triplicate. After $48 \mathrm{~h}$, non-adherent apoptotic/dead cells and viable adherent cells were collected from each well and centrifuged at $310 \mathrm{xg}$ for $5 \mathrm{~min}$ at $4^{\circ} \mathrm{C}$. Cell pellets were resuspended in growth media, diluted in Guava ${ }^{\circledR}$ ViaCount $^{\circledR}$ reagent (Guava Technologies, Hayward, CA) and analyzed after 5 min incubation with a Guava personal cytometer using the Guava CytoAnalysis software. The amount of live/ healthy cells, apoptotic and dead cells were expressed as a

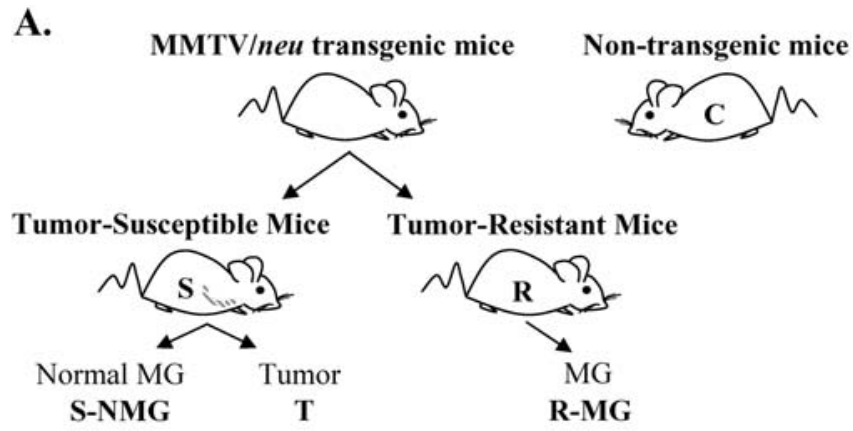

B.

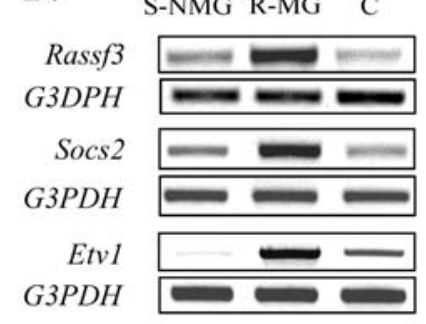

\section{C.}

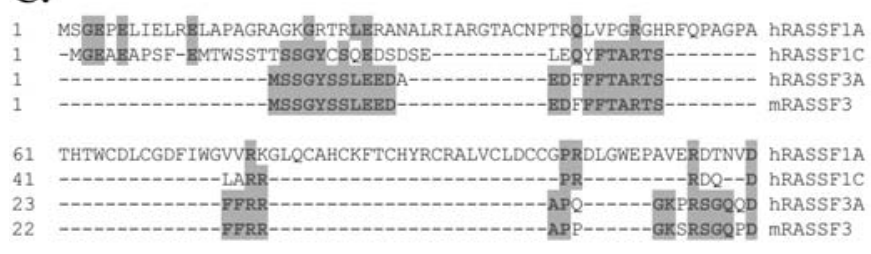

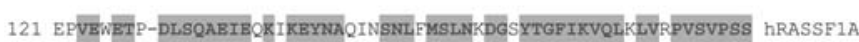

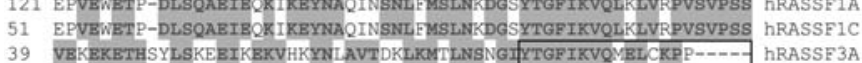
39
38
38

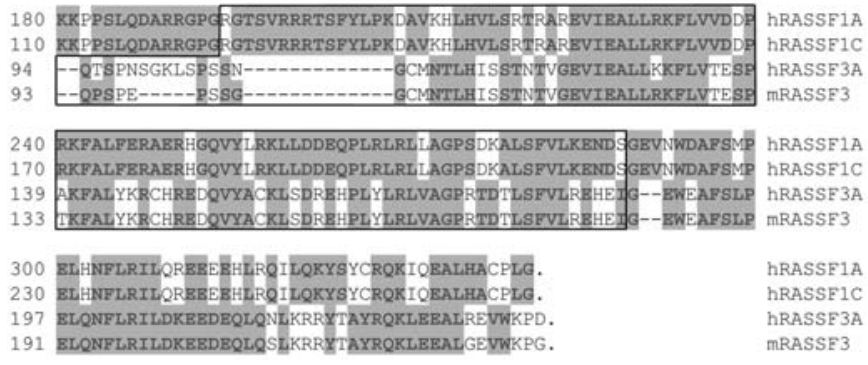

Figure 1. Nomenclature of mouse groups and microarray data confirmation for selected genes by RT-PCR analysis. (A) Three groups of virgin female mice were defined as follows: 1) Tumor-susceptible MMTV/neu transgenic mice that developed mammary tumors (S), 2) Tumor-resistant MMTV/neu transgenic mice that did not develop tumors (R) and 3) non-transgenic FVB mice used as control (C). Mammary gland isolated from the $\mathrm{S}$ group is defined as susceptible normal mammary gland (S-NMG). (B) Total RNA was isolated from the mammary glands of three 11-month-old $\mathrm{R}$ and $\mathrm{S}$ mice and of three age-matched $\mathrm{C}$ mice, then pooled into one sample per mouse group to conduct RT-PCR analyses for Rassf3, Socs2, Etv1 genes and $G 3 P D H$ as a loading control. The gels shown from pooled samples are representatives of individual mammary gland samples. (C) The alignment of hRASSF1A (GenBank NM_007182), hRASSF1C (GenBank NM_170713), hRASSF3 (GenBank BC100951) and mRASSF3 (GenBank NM_138956) were generated with the MegAlign program from Lasergene (DNASTAR) using the ClustalW algorithm. Identical amino acids are highlighted in gray and boxes define the RA domains.

percentage of the total cellular population for each sample. Data are expressed as mean \pm SEM and are representative of two experiments. 

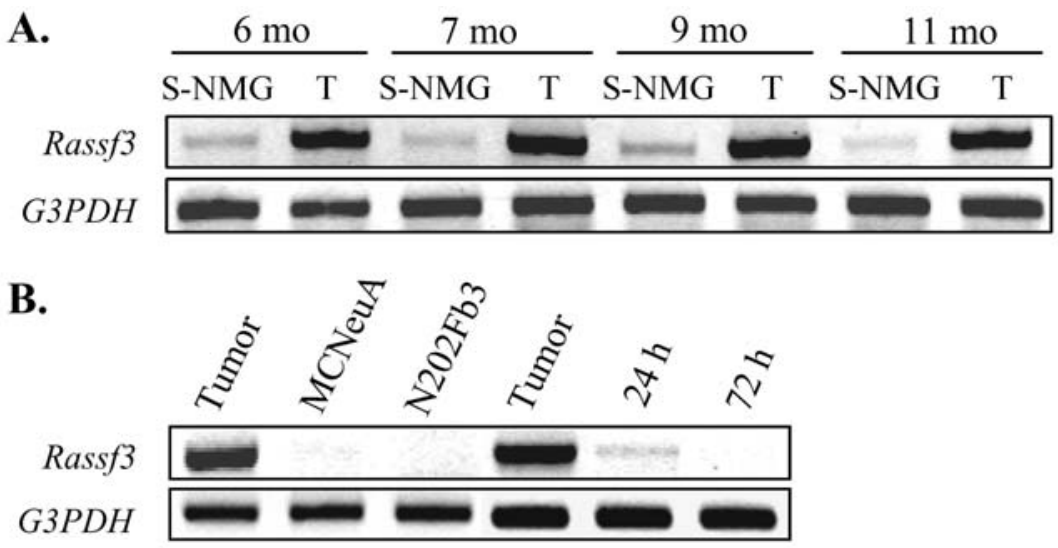

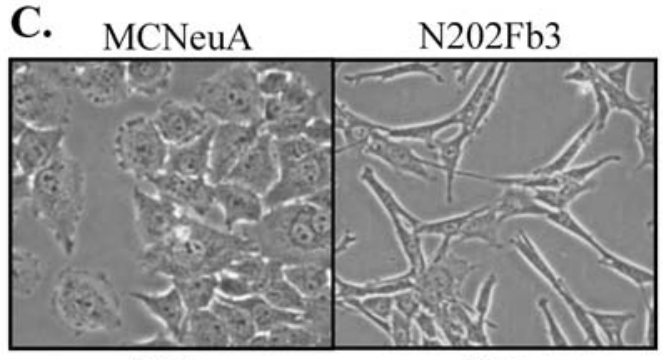

$24 \mathrm{~h}$ $72 \mathrm{~h}$

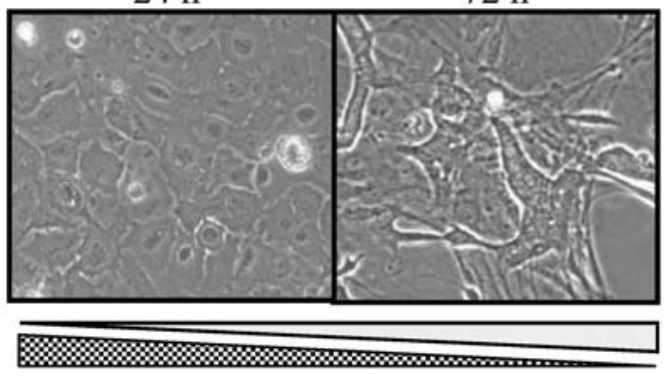

\section{Results}

Identification and confirmation of differentially expressed genes by cDNA microarray analysis. The approach utilized sought for genes that exhibited an opposing pattern of expression between the mammary glands of tumor-resistant mice (R-MG) and the mammary glands of tumor-susceptible mice (S-NMG) (Fig. 1A). Particular attention was accorded to genes up-regulated in R-MG but down-regulated in S-NMG (Table I). We focused on genes involved in signal transduction and on genes which could interact with proteins involved in the HER2/neu receptor tyrosine kinase signaling pathway. Three genes were selected to confirm microarray data by semi-quantitative RT-PCR analyses on total RNA samples isolated from mammary glands of the three groups of mice. Two of these genes encode for intracellular proteins involved in signal transduction and correspond to the Socs 2 gene, a well-characterized suppressor of cytokine signaling (20), and the novel Rassf3 gene which belongs to the Ras association domain family (RalGDS/AF-6) gene (21). The third gene is Etv1 which belongs to the ETS family of transcription factors (22). The Rassf3, Socs2 and Etv1 genes are overexpressed in mammary glands of tumor-resistant mice (R-MG) compared to tumor-susceptible mice (S-NMG) and control mice (C), which confirmed the microarray data (Fig. 1B). Rassf3 expression level was also validated by RT-PCR in individual
Figure 2. Rassf3 expression is higher in mammary tumors than in adjacent normal mammary tissues but absent in HER2/neu mammary tumor-derived cells. (A) Total RNA was isolated from pairs of mammary tumors (T) and adjacent normal tissues (S-NMG) of MMTV/neu transgenic mice that developed tumors at the age of 6, 7,9 and 11 months (mo) to conduct RTPCR analyses with appropriate primers for Rassf3 and G3PDH as a loading control. (B) Total RNA was isolated from a primary HER2/neu mouse mammary tumor tissue (T), from established epithelial cells (MCNeuA) and fibroblasts (N202F3) derived from a HER2/neu mouse mammary tumor and from the primary cultured cells of a HER2/neu mammary tumor mix at $24 \mathrm{~h}$ (passage 1) and $72 \mathrm{~h}$ (passage 3 ) for RT-PCR analyses. (C) The cell morphology of MCNeuA and N202F3 cells and of primary cultured cells at 24 and $72 \mathrm{~h}$ post-passages was recorded under a microscope to demonstrate the different cell populations (F, fibroblast; E, epithelium).

mammary glands from 11-month-old tumor-resistant mice (R-MG) and tumor-susceptible mice (S-NMG) from a different set of mice. Rassf3 was up-regulated in 3 out of 5 individual R-MG mammary glands but totally absent in individual S-NMG mammary glands (5 out of 5) (data not shown).

Shared homology between RASSF family members. Sequences were aligned with ClustalW algorithm (Fig. 1C). The mouse (m) RASSF3 (232 amino acids; M.W. 26.7 kDa) and human (h) RASSF3 (247 amino acids; M.W. $28.6 \mathrm{kDa}$ ) sequences share $94 \%$ identity at the amino acid level, whereas the hRASSF1A (340 amino acids) and hRASSF1C (270 amino acids) isoforms share $81 \%$ identity at the amino acid level and differ mainly at the N-terminus. The mRASSF3 and hRASSF3 both share $\sim 40 \%$ and $44 \%$ identity with hRASSF1A and hRASSF1C proteins, respectively. The proteins contain a highly conserved Ras association (RalGDS/AF-6) (RA) domain at the $\mathrm{C}$-terminus defined by the boxes in Fig. $1 \mathrm{C}$.

Rassf3 expression is higher in mammary tumor than in normal adjacent tissue. Total RNA from four pairs of mammary tumors (T) and adjacent normal tissues (S-NMG) of MMTV/ neu mice that developed tumors at the age of $6,7,9$ or 11 months was used for RT-PCR analyses to study the expression patterns of Rassf3 (Fig. 2A). Surprisingly, Rassf3 expression levels were consistently higher in mammary tumors $(\mathrm{T})$ than 
Table I. cDNA microarray analysis. ${ }^{a}$

Signal transduction

Growth factors and growth factor receptors

Mia Melanoma inhibitory activity 1

Tm4sf3

Tetraspanin 8

NM_019394

Transcription factors

Cited4

Cbp/p300-interacting transactivator,

BC025461

\section{Etv1} with Glu/Asp-richcarboxy-terminal domain, 4

BC025116

Tcfap2c

Ets variant gene 1

Etv5

Transcription factor AP-2, $\gamma$

NM_007960

BC003778

Hey 1

Ets variant gene 5

BG966751

Intracellular signaling molecules

\section{Socs2}

Rassf3

Suppressor of cytokine signaling 2

Ras association (RalGDS/AF-6) domain family 3

Cell tissue structure

Cytoskeleton

Mylpc

Tnce

Tpm3

Homer2

Tnnt1

Myh7

Myh2

Tnni1
Mylc
Myosin, light polypeptide 2, regulatory, cardiac, slow Troponin C, cardiac/slow skeletal

Tropomyosin 3, $\gamma$

Homer homolog 2 (Drosophila)

Troponin T1, skeletal, slow

Myosin, light polypeptide 3

Myosin, heavy polypeptide 7 , cardiac muscle, $\beta$

Myosin, heavy polypeptide 2 , skeletal muscle, adult Troponin I, skeletal, slow 1
NM_007706

BC011511

NM_010861

NM_009393

NM_022314

AB017136

NM_011618

X67685

NM_080728

BC008538

NM_021467

NM_007740

NM_007729

NM_007675

AW553512

NM_016685

NM_011926

BC005747

BM208112

BI788452

Papilin, proteoglycan-like sulfated glycoprotein

Sperm associated antigen 5

Tissue inhibitor of metalloproteinase 4

Timp4

Treansporter

Electron transporter

2410043F08Rik

Filamin binding LIM protein 1

NM_133754

Potassium intermediate/small conductance calcium-activatedchannel, subfamily N, member 4

Kenn4

Kcnk1 Potassium channel, subfamily K, member 1

NM_008433

NM_008430

Other transporter

Aqp5

Slc29a1

\section{Aquaporin 5}

Solute carrier family 29 (nucleoside transporters), member 1

Lman1

Lectin, mannose-binding, 1

NM_009701 
Table I. Continued.

Metabolism
Fatty acid biosynth
Scd2
B4galt6
9430020A05Rik
Glycolysis
Aldo3
Hk1
Amino acid metabo
Got1
Other
2310047E01Rik
Egln3
4930555L11Rik
Cp
Rdh12
Ckmt2
Other genes
Xlr3a
Scrg1
- - -
4931430I01Rik
- - -
Emu1-pending

Metabolism

Fatty acid biosynthesis

Scd2

9430020A05Rik

Stearoyl-Coenzyme A desaturase 2

$\begin{array}{ll}\text { BG060909 } & 2.0 \\ \text { BG066773 } & 2.0 \\ \text { BI153391 } & 2.0 \\ & \\ \text { BM941201 } & 3.6 \\ \text { NM_010438 } & 3.0\end{array}$

Aldolase 3, C isoform

Hexokinase 1 polypeptide 6

Acyl-CoA synthetase long-chain family member 4

NM_010438

Glutamate oxaloacetate transaminase 1, soluble

AA792094

Carbonic anyhydrase 12

EGL nine homolog 3 (C. elegans)

Ethanolamine kinase 1

Ceruloplasmin

Retinol dehydrogenase 12

Creatine kinase, mitochondrial 2

$\begin{array}{ll}\text { AK009873 } & 3.0 \\ \text { NM_028133 } & 2.8 \\ \text { BG066916 } & 2.4 \\ \text { BB531328 } & 2.3 \\ \text { BC016204 } & 2.1 \\ \text { AK009042 } & 2.1\end{array}$

X-linked lymphocyte-regulated 3a

NM_011726

3.6

Scrapie responsive gene 1

Similar to Shb-like adapter protein, Shf - human

NM_009136

3.4

EF hand domain containing 1

BB798279

2.4

BC019531

2.2

Tumor differentially expressed 1

BM239368

2.1

EMI domain containing 1

NM_080595

${ }^{\text {aList }}$ of genes that are overexpressed in the mammary gland of tumor-resistant MMTV/neu female transgenic mice compared to tumorsusceptible MMTV/neu littermates.

in adjacent normal mammary tissues (S-NMG), independent of age at which the tumor appeared. Sequencing analyses of the Rassf3 gene cloned from 6 mammary tumors revealed no consensus mutation (data not shown). In addition, this particular expression pattern of the Rassf 3 gene between $\mathrm{T}$ and S-NMG tissues was not observed for other members of the Rassf family, such as Rassf1, Rassf2, Rassf4 and Rassf5 (data not shown).

To further study the expression pattern of Rassf3 within tumor tissues, expression levels were compared between epithelial cells (MCNeuA) and fibroblasts (N202Fb3), both derived from a Her2/neu mammary tumor (23). Interestingly, even though Rassf 3 expression was high in Her2/neu primary tumors, Rassf 3 expression appeared to be absent in the two established cell lines (Fig. 2B). Rassf3 expression pattern in the primary cultured cells from a Her2/neu mammary tumor was also examined at different time points post-passage (24 and $72 \mathrm{~h}$ ). Rassf3 gene was expressed in primary cells at early time points ( $24 \mathrm{~h}$ ), but its expression diminished after $72 \mathrm{~h}$ (Fig. 2B). Concurrently, the morphology of the primary culture revealed a switch in cell population composition, corresponding to an increase in fibroblast $(\mathrm{F})$ population and a progressive loss of epithelial cells (E) population (Fig. 2C).
RASSF3 expression is inversely correlated to HER2 expression in human breast cancer cell lines. Ten human breast cancer cell lines were analyzed for HER2 and phosphorylated HER2 (p-HER2) protein levels and for RASSF3 gene expression level. RASSF3 gene expression was inversely correlated to HER2 protein levels among the 10 cell lines (Fig. 3A). $R A S S F 3$ expression was minimal or non-detectable in SKBR-3 and BT-474 cell lines, which express high levels of HER2 and p-HER2. On the contrary, RASSF3 expression levels were high in MDA-MB-231 and MDA-MB-436 cell lines, which did not show expression of HER2 protein. T47D cells showed moderate levels of both endogenous RASSF3 and HER2.

RASSF3 expression in human tissues is tumor-type specific. To assess RASSF3 gene expression in human tumors compared to adjacent normal tissues, total RNA samples from five pairs of human tumors and adjacent normal tissues from breast, lung, uterus, colon and cervix were used for RT-PCR analysis (Fig. 3B). RASSF3 expression was higher in tumor compared to adjacent tissue of the breast and cervix samples $(\mathrm{p}<0.05)$. RASSF 3 expression level was lower in tumor compared to adjacent tissue of lung, uterus and colon organs. These 
A.

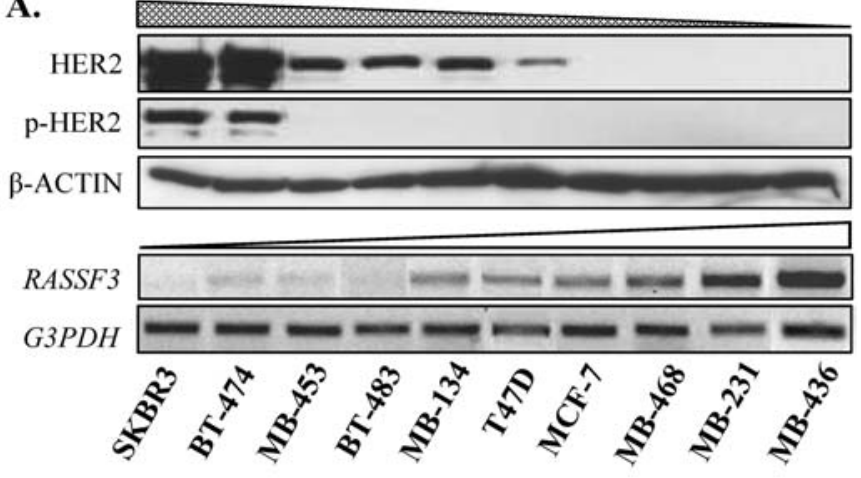

B.

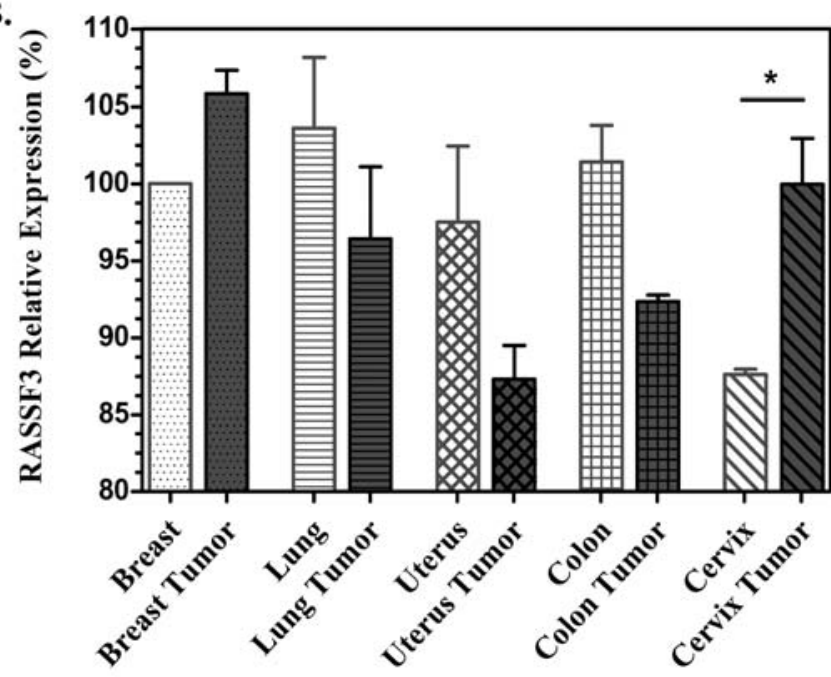

Figure 3. RASSF3 expression is inversely correlated to HER2 expression levels and is tumor-type specific. (A) HER2 and p-HER2 protein levels, determined by immunoblot analyses, were compared with levels of RASSF3 mRNA, determined by RT-PCR analyses, in 10 human breast cancer cells. $ß$-actin and G3PDH were used as loading controls. (B) RASSF3 mRNA levels were determined in pairs of tumor and adjacent normal tissue from human breast, lung, uterus, colon and cervix, by RT-PCR analysis. G3PDH was used as a loading control. Kodak 1D image analysis software was used to compare the mean intensity of the bands. Mean intensity was set to $100 \%$ for normal breast tissue. Data are presented as percentage and correspond to RASSF3 relative expression in the different tissues. The data represent the mean \pm SEM of two independent experiments $\left({ }^{*} \mathrm{p}<0.05\right)$.

observations indicate that $R A S S F 3$ expression patterns are tumor-type specific.

RASSF3 expression reduces cell viability and induces apoptosis in human breast cancer cell lines. Transient transfection was used to study the effects of Rassf3 in vitro. SKBR3 human breast cancer epithelial cell line, which expresses very high levels of HER2 protein, was selected to examine the effects of Rassf3 expression on cell viability. Rassf 3 expression reduced cell viability in a dose-dependent manner. The inhibitory effect reached a maximum with $2 \mu \mathrm{g}$ of pcDNA3.1/ Rassf3 plasmid and after $24 \mathrm{~h}$ of proliferation. Multiple epithelial cell lines (SKBR3, BT-474, MCNeuA and HC11), which express high levels of HER2 protein, were used to confirm Rassf3 inhibitory effect. Cells were transfected with $2 \mu \mathrm{g}$ of pcDNA3.1/Rassf3 plasmid or vector and cellular viability was measured after $24 \mathrm{~h}$. For this transfection experiment, a novel polyclonal antibody was developed against RASSF3. The specificity was confirmed with a titer test and by immunoblot with varied amounts of RASSF3 protein (data not shown) both test revealing the specificity of the antibody and the ability to distinguish variable amounts of RASSF3. RASSF3 protein expression was confirmed post-transfection in each transfected cell line (Fig. 4A). Rassf3 transient expression reduced cell viability by $20 \%$ in SKBR3, $8 \%$ in BT-474, 15\% in MCNeuA and 10\% in HC11 cells (Fig. 4B).

The effects of RASSF3 and H-RAS on cell viability were compared by transfection with activated $H-R A S$ [pcDNA3.1/ $\mathrm{H}-R A S(G 12 \mathrm{~V})$ ] or dominant-negative $H$-RAS [pcDNA3.1/ $\mathrm{H}-R A S(S 17 N)]$ in SKBR3 cells. Rassf3 reduced cell viability in a highly significant manner compared to activated H-RAS or dominant-negative H-RAS alone $(\mathrm{p}<0.01)$ which had minimal effects on cell viability (Fig. 4C).

To determine the potential mechanism behind the growthinhibitory properties of Rassf3, Guava ViaCount ${ }^{\mathrm{TM}}$ assay for cellular viability were performed on transfected SKBR3 cells (Fig. 4D). SKBR3 cells were transfected with 2 or $4 \mu \mathrm{g}$ of vector and pcDNA3.1/Rassf3 and collected after 48 h. Rassf3 transfection caused $\sim 15-20 \%$ increase in the number of apoptotic and dead cells compared to non-specific vector transfection. These changes in cells numbers were statistically highly significant $(\mathrm{p}<0.01)$ with 2 or $4 \mu \mathrm{g}$ of pcDNA3.1/Rassf3 plasmid.

MMTV/Rassf3-neu bi-transgenic mice display delayed tumor formation. To study Rassf 3 function in vivo, two novel transgenic mouse lines were generated: MMTV/Rassf3 transgenic and MMTV/Rassf3-neu bi-transgenic lines. Incorporation of Rassf3 transgene was confirmed by PCR analysis on genomic DNA isolated from mouse tail biopsies (Fig. 5A). Tissues from female MMTV/Rassf3 mice and their non-transgenic littermates were analyzed by RT-PCR for Rassf3 gene expression which indicated that the Rassf3 gene is expressed in the mammary gland as well as in the brain, small intestine and muscle of MMTV/Rassf 3 transgenic mice (data not shown). RASSF3 protein was not detected in mammary gland tissues of MMTV/Rassf3 mice and MMTV/ Rassf3-neu bi-transgenic mice by immunoblot, although Rassf3 mRNA was present and amplified by RT-PCR analysis of mammary gland homogenates (Fig. 5B). However, RASSF3 protein was detected in the brain tissue of all MMTV/Rassf3 transgenic mice and of MMTV/Rassf3-neu bi-transgenic mice whereas it was not detected in littermates (Fig. 5B).

To assess the effect of Rassf3 overexpression on HER2/neu mammary tumorigenesis, MMTV/Rassf3-neu transgenic mice and MMTV/ne $u^{+-}$littermates were monitored for development of palpable mammary tumors. Comparison of mammary tumor incidence revealed a delay in tumor formation $(\mathrm{p}=0.0552)$ in bi-transgenic mice (Fig. 5C). The $\mathrm{t}_{50}$ of MMTV/Rassf3-neu bi-transgenic mice $\left(n=37 ; t_{50}=262\right)$ was increased by 37 days compared to the $\mathrm{t}_{50}$ of MMTV/ne $u^{+/-}$littermates $(\mathrm{n}=32$; $\left.\mathrm{t}_{50}=225\right)$. RASSF3 protein was detected by immunoblot in tumors of MMTV/Rassf3-neu bi-transgenic mice and of

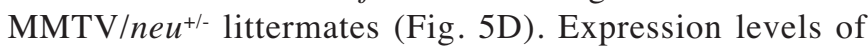
RASSF3 and p-HER2 proteins appeared to be both higher in tumors from bi-transgenic mice than in tumors from littermates. 

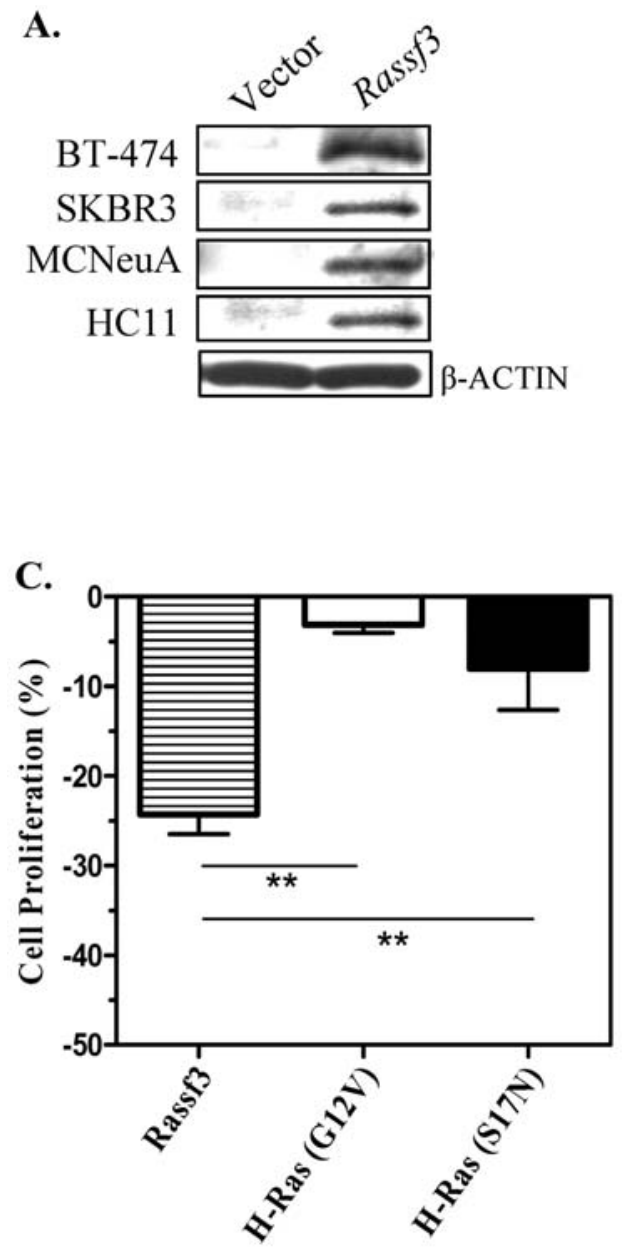
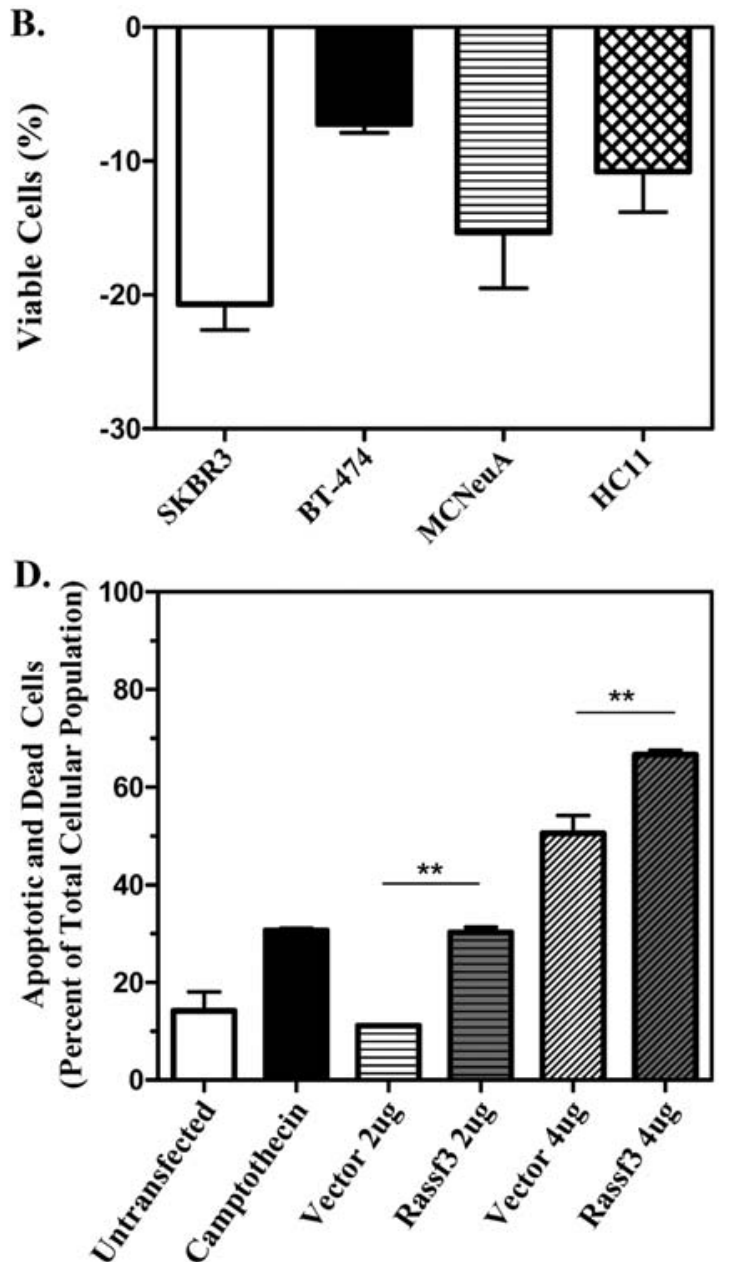

Figure 4. Rassf3 expression reduces cell viability and induces cell death in SKBR3 human breast cancer cells. (A) RASSF3 protein expression was confirmed by immnoblot analysis using a specific anti-RASSF3 antibody in four cell lines (SKBR3, BT-474, MCNeuA and HC11) expressing high levels of HER2 protein, $24 \mathrm{~h}$ after transient transfection with $2 \mu \mathrm{g}$ of Rassf3 or vector plasmids. B-actin was used as a loading control and is representative of all the gels. (B) Forty-eight hours post-transfection, cells were plated at an equal cell concentration and viability was monitored after $24 \mathrm{~h}$. Data are presented as mean \pm SEM of a percentage of Rassf3 inhibition compared to vector. Means are obtained from 8 independent experiments for SKBR3 cells and 3 independent experiments for BT-474, MCNeuA and HC11 cells. (C) SKBR3 cells were transfected with $2 \mu \mathrm{g}$ of vector, pcDNA3.1/Rassf3, pcDNA3.1/H-RAS (G12V) or pcDNA3.1/ $H-R A S(S 17 N)$. Cells were plated $48 \mathrm{~h}$ post-transfection at an equal cell concentration and viability was monitored after $24 \mathrm{~h}$. Data are presented as a percentage of Rassf3, $H$-RAS $(G 12 \mathrm{~V})$ and $H$-RAS (S17N) effect on cell viability compared to vector. The data correspond to the mean \pm SEM of percentages obtained from 4 independent experiments $\left(^{* *} \mathrm{p}<0.01\right)$. (D) SKBR3 cells were transiently transfected with 2 or $4 \mu \mathrm{g}$ of vector and Rassf3 plasmid and collected after $48 \mathrm{~h}$ to run the Guava ViaCount ${ }^{\mathrm{TM}}$ assay. Untransfected cells and camptothecin-treated cells were used as negative and positive controls respectively. The amount of apoptotic and dead cells were expressed as a percentage of the total cellular population for each treatment. Data are presented as mean \pm SEM of triplicates and are representative of two separate experiments $\left({ }^{* *} \mathrm{p}<0.01\right)$.

\section{Discussion}

The novel approach described herein, utilizing MMTV/neu transgenic mouse model, focused on naturally acquired tumorresistant MMTV/neu female transgenic mice which either have a significant delay in or resistance to neu tumorigenesis. This approach identified Rassf 3 as a candidate tumor suppressor gene which may be involved in this resistance to or the delaying of HER2/neu-initiated mammary tumorigenesis. Our data demonstrated that the Rassf3 gene is overexpressed in the mammary gland of tumor-resistant MMTV/ neu mice compared to tumor-susceptible MMTV/neu transgenic littermates or non-transgenic FVB mice (Fig. 1B). The RASSF 3 gene is classified as a member of the Ras association domain (RalGDS/AF-6) family gene (RASSF), which contains characterized RAS effectors and tumor suppressor genes (15-18). However, the Rassf3 gene remains relatively uncharacterized and no functional studies of human RASSF 3 and mouse Rassf3 genes have been reported to date.

It has been documented that some genes with tumor suppressor activity, notably TGF- $\beta, \mathrm{p} 53$ and SOCS tumor suppressors, show a marked increase in their expression in specific cancers $(20,24-26)$. In accordance with this observation, our data indicate that the Rassf 3 gene is significantly up-regulated in neu-specific mouse mammary tumors compared to adjacent normal tissues. Overexpression of Rassf3 gene in mouse mammary tumors may be the result of a cellular defensive response to high levels of HER2/Neu proteins in mammary tumors of MMTV/neu transgenic mice (Fig. 2A).

Comparison of HER2 protein and RASSF3 mRNA levels among multiple human breast cancer cell lines revealed an 
A.

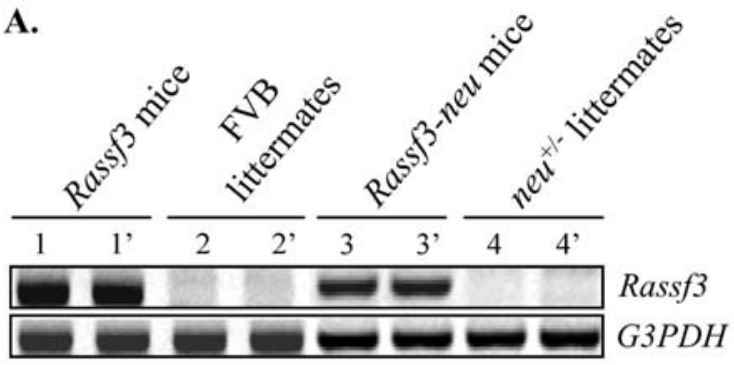

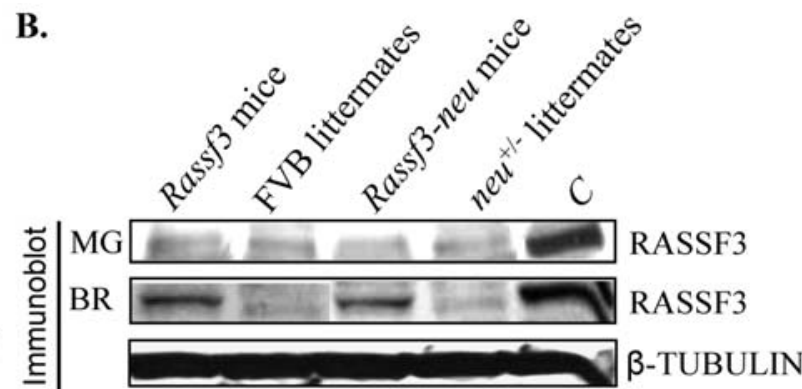

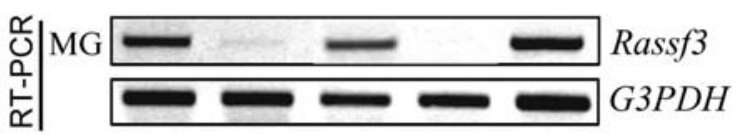

C.
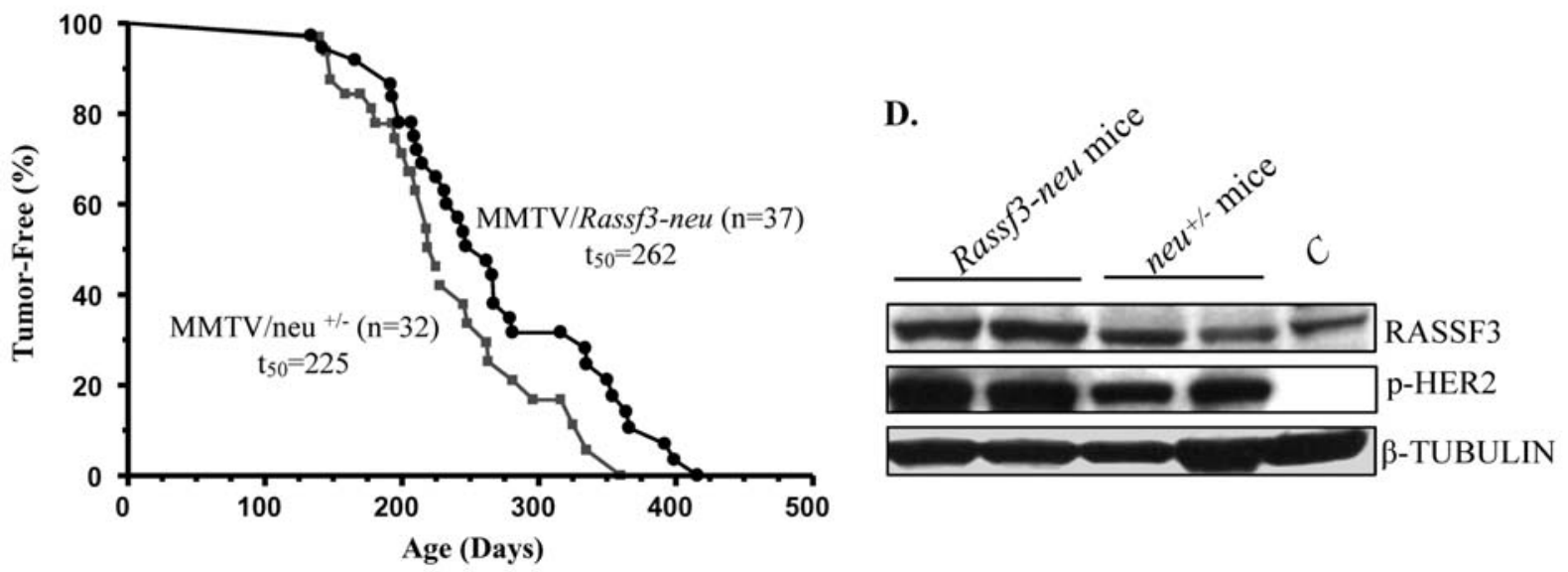

Figure 5. Bi-transgenic MMTV/Rassf3-neu mice display increased tumor latency. (A) Transgene incorporation was confirmed by PCR analysis of genomic tail DNA from MMTV/Rassf3 transgenic mice (1, 1'), FVB non-transgenic littermates (2, 2'), MMTV/Rassf3-neu bi-transgenic mice (3, 3') and MMTV/neu ${ }^{+/}$ littermates $\left(4,4^{\prime}\right)$ with a pair of specific primers that span the MMTV promoter and the Rassf 3 cDNA sequence. G3PDH was used as a loading control. (B) Tissue lysates were isolated from mammary gland and brain tissues of transgenic mice and their littermates for immunoblot analysis with an anti-RASSF3 antibody and total RNA samples from mammary gland tissues were used for RT-PCR analysis. Purified RASSF3 protein was used as a positive control (C). ß-tubulin and G3PDH were used as loading controls. (C) Data are plotted as the percentage of tumor-free female mice (y-axis) as a function of age in days (x-axis). MMTV/Rassf3-neu bi-transgenic mice $(\mathrm{n}=37)$ and $\mathrm{MMTV} /$ ne $^{+/-}$littermates $(\mathrm{n}=32)$ are represented by closed circles and closed squares, respectively. The $\mathrm{t}_{50}$ of MMTV/Rassf3-neu bi-transgenic mice and their littermates correspond to 262 and 225 days, respectively. Log rank test, $\mathrm{p}=0.0552$. (D) Protein lysates were isolated from mammary tumors of MMTV/Rassf3-neu bi-transgenic mice and of MMTV/neut/- littermates for immunoblot analysis with anti-RASSF3 and anti-phospho-HER2 antibodies. Purified RASSF3 protein was used as a positive control (C) and B-tubulin as a loading control.

inverse correlation of expression between these two genes (Fig. 3A). The inverse correlation of expression between an oncogene and a tumor suppressor gene has been reported in different cancers (15). The inverse correlation suggests that HER 2 and RASSF3 genes have a cooperative role in breast carcinogenesis and that they are involved in the same pathway. The analysis of human tumors and adjacent normal tissues also revealed that RASSF3 overexpression pattern is dependent on the tissue and cancer types (Fig. 3B). Therefore, Rassf3 overexpression may become an interesting biomarker in breast cancer.

Most HER2/neu mammary tumors in this study contain high levels of Rassf3 mRNA (Fig. 2A). Since all tumor samples prepared in this study were tissue homogenates, it is impossible to differentiate the origin of Rassf 3 expression in tumors. The primary culture experiment suggests that Rassf 3 expression in the mouse HER2/neu tumor mix is likely from the epithelium cells rather than the stromal cells since Rassf3 expression level decreased drastically within days when the population of epithelial cells dropped while the stromal cell population increased after passages (Fig. 2C). The loss of Rassf3 expression can be explained by the epithelialmesenchymal transition hypothesis, which is often characterized by the turning-off of genes encoding epithelial markers (e.g. E-cadherins), the loss of epithelial features and the increase in markers of mesenchymal cells. However, we have noted that Rassf3 expression in established epithelium cells, derived directly from the MMTV/neu mouse tumor (Fig. 2B), is barely detectable, which is consistent with the results shown in Fig. 3A where Rassf3 level is inversely correlated with HER2 level. One possible explanation is that it is well known that breast cancer cells are genetically unstable. Once extracted from the tumor, they can undergo specific genotype/phenotype alterations resulting from long- 
term culture in simplified conditions. Nonetheless, more studies are needed to evaluate whether Rassf 3 expression in tumors could be used as a valuable marker to predict the prognoses.

Ectopic expression of Rassf3 reduced cell viability of various cell lines including SKBR3 and BT474 human breast cancer cell lines, MCNeuA mouse cancer cell line and HC11 mouse cell line (Fig. 4B), all of which are characterized by expression of high levels of HER2 protein. The growthinhibitory effect of Rassf 3 was compared to an activated form of H-RAS and a dominant negative form of H-RAS in SKBR3transfected cells. It has been shown that differences in effector binding and signaling exist between the different RAS isoforms for the RASSF proteins $(8,27)$; therefore, we used the H-RAS isoform that has been shown to specifically interact with RASSF1, since RASSF3 gene shares more homology with this member of the RASSF family. Rassf3 growth-inhibition was greater than that of the dominant negative form of H-RAS response in SKBR3-transfected cells (Fig. 4C). Investigation of the mechanism by which Rassf3 promotes growth inhibition in SKBR3 cells suggested that Rassf3 promotes cell apoptosis (Fig. 4D) which supports a role for Rassf3 as a tumor suppressor gene.

To date, in the RASSF family, only RASSF1A animal model was reported (28). A mouse knockout for Rassfla, where the Rassfla was specifically inactivated, revealed that Rassfla ${ }^{+/-}$and Rassfla $a^{-/}$were prone to spontaneous tumorigenesis with advanced age (18-20 months) and were more susceptible to chemical carcinogen-induced tumor formation. The tumors included lung adenomas, lymphomas and breast carcinomas. These data reinforced the role of RASSF1 gene as a tumor suppressor. In this study, to elucidate the role of Rassf3 in HER2/neu-initiated breast cancer, MMTV/Rassf3 transgenic mice were cross-bred with MMTV/neu transgenic mice to produce MMTV/Rassf3-neu bi-transgenic mouse line. This novel MMTV/Rassf3-neu bi-transgenic mouse model permitted the determination of Rassf 3 influence on HER2/neu mammary tumor formation in vivo. Despite the fact that RASSF3 protein in our established MMTV/Rassf3 transgenic mouse line was detected mainly in the brain tissue but minimally present in the mammary gland, Rassf3 expression still delayed tumor onset in the bi-transgenic mouse line (Fig. 5C). We believe that if RASSF3 expression levels and activity were increased in the mammary gland of the bi-transgenic mouse line, the delay in tumor onset would have been more significant. It is interesting to note that RASSF3 and p-HER2 protein levels in mammary tumors of MMTV/Rassf3-neu bi-transgenic mice and MMTV/neu ${ }^{+/}$ littermates are positively correlated (Fig. 5D). We speculate that high levels of RASSF3 found in tumors are not the causal factor but are the result of a cellular/host defensive response. It appears that RASSF3 expression is induced in response to HER2 activity in HER2/neu-positive tumors.

In summary, this study presented a novel approach for studying the well-documented MMTV/neu transgenic mouse model by focusing on naturally acquired tumor-resistant MMTV/neu female transgenic mice. We provide evidence suggesting an important role for the Rassf3 gene in the process of HER2/neu-initiated mammary tumorigenesis. Furthermore, we report the first functional study for the Rassf3 gene using in vitro and in vivo models. The findings suggest that the Rassf3 gene exhibits the properties of a RAS effector and tumor suppressor gene. However, the molecular mechanism of growth-inhibition of the Rassf3 gene and its particular role in HER2/neu tumor initiation and progression needs further investigation.

\section{References}

1. Slamon DJ, Clark GM, Wong SG, Levin WJ, Ullrich A and McGuire WL: Human breast cancer: correlation of relapse and survival with amplification of the HER-2/neu oncogene. Science 235: 177-182, 1987

2. Slamon DJ, Godolphin W, Jones LA, et al: Studies of the HER-2/ neu proto-oncogene in human breast and ovarian cancer. Science 244: 707-712, 1989.

3. Guy CT, Webster MA, Schaller M, Parsons TJ, Cardiff RD and Muller WJ: Expression of the neu protooncogene in the mammary epithelium of transgenic mice induces metastatic disease. Proc Natl Acad Sci USA 89: 10578-10582, 1992.

4. Lodish H BD, Berk A, Zipursky SL, Matsudaira P: Molecular Cell Biology. W.H. Freeman and Co., New York, 2001.

5. Bazley LA and Gullick WJ: The epidermal growth factor receptor family. Endocr Relat Cancer 12 (Suppl 1): 17-27, 2005.

6. Clark GJ and Der CJ: Aberrant function of the Ras signal transduction pathway in human breast cancer. Breast Cancer Res Treat 35: 133-144, 1995.

7. von Lintig FC, Dreilinger AD, Varki NM, Wallace AM, Casteel DE and Boss GR: Ras activation in human breast cancer. Breast Cancer Res Treat 62: 51-62, 2000.

8. Rodriguez-Viciana P, Sabatier C and McCormick F: Signaling specificity by Ras family GTPases is determined by the full spectrum of effectors they regulate. Mol Cell Biol 24: 4943-4954, 2004.

9. Lowy DR, Johnson MR, DeClue JE, et al: Cell transformation by ras and regulation of its protein product. Ciba Found Symp 176: 67-80, 1993.

10. Chen CY and Faller DV: Direction of p21ras-generated signals towards cell growth or apoptosis is determined by protein kinase $\mathrm{C}$ and $\mathrm{Bcl}-2$. Oncogene 11: 1487-1498, 1995.

11. Chen CY, Liou J, Forman LW and Faller DV: Differential regulation of discrete apoptotic pathways by Ras. J Biol Chem 273: 16700-16709, 1998 .

12. Khokhlatchev A, Rabizadeh S, Xavier R, et al: Identification of a novel Ras-regulated proapoptotic pathway. Curr Biol 12: 253-265, 2002.

13. Shao J, Sheng H, DuBois RN and Beauchamp RD: Oncogenic Ras-mediated cell growth arrest and apoptosis are associated with increased ubiquitin-dependent cyclin D1 degradation. J Biol Chem 275: 22916-22924, 2000.

14. Cox AD and Der CJ: The dark side of Ras: regulation of apoptosis. Oncogene 22: 8999-9006, 2003.

15. Agathanggelou A, Cooper WN and Latif F: Role of the Rasassociation domain family 1 tumor suppressor gene in human cancers. Cancer Res 65: 3497-3508, 2005.

16. Eckfeld K, Hesson L, Vos MD, Bieche I, Latif F and Clark GJ: RASSF4/AD037 is a potential ras effector/tumor suppressor of the RASSF family. Cancer Res 64: 8688-8693, 2004.

17. Hesson L, Dallol A, Minna JD, Maher ER and Latif F: NORE1A, a homologue of RASSF1A tumour suppressor gene is inactivated in human cancers. Oncogene 22: 947-954, 2003.

18. Vos MD, Ellis CA, Elam C, Ulku AS, Taylor BJ and Clark GJ: RASSF2 is a novel K-Ras-specific effector and potential tumor suppressor. J Biol Chem 278: 28045-28051, 2003.

19. Wagner TE, Hoppe PC, Jollick JD, Scholl DR, Hodinka RL and Gault JB: Microinjection of a rabbit beta-globin gene into zygotes and its subsequent expression in adult mice and their offspring. Proc Natl Acad Sci USA 78: 6376-6380, 1981.

20. Raccurt M, Tam SP, Lau P, et al: Suppressor of cytokine signalling gene expression is elevated in breast carcinoma. $\mathrm{Br} \mathrm{J}$ Cancer 89: 524-532, 2003.

21. Tommasi S, Dammann R, Jin SG, Zhang Xf XF, Avruch J and Pfeifer GP: RASSF3 and NORE1: identification and cloning of two human homologues of the putative tumor suppressor gene RASSF1. Oncogene 21: 2713-2720, 2002. 
22. Goel A and Janknecht R: Concerted activation of ETS protein ER 81 by p160 coactivators, the acetyltransferase p300 and the receptor tyrosine kinase HER2/Neu. J Biol Chem 279: 14909-14916, 2004.

23. Campbell MJ, Wollish WS, Lobo M and Esserman LJ: Epithelial and fibroblast cell lines derived from a spontaneous mammary carcinoma in a MMTV/neu transgenic mouse. In Vitro Cell Dev Biol Anim 38: 326-333, 2002.

24. Oft M, Heider $\mathrm{KH}$ and Beug $\mathrm{H}$ : TGFbeta signaling is necessary for carcinoma cell invasiveness and metastasis. Curr Biol 8: 1243-1252, 1998.
25. Gonzalez-Palacios F, Sancho M, Martinez JC and Bellas C: Microvessel density, p53 overexpression, and apoptosis in invasive breast carcinoma. Mol Pathol 50: 304-309, 1997.

26. Rowland BD and Peeper DS: KLF4, p21 and context-dependent opposing forces in cancer. Nat Rev Cancer 6: 11-23, 2006.

27. Yan J, Roy S, Apolloni A, Lane A and Hancock JF: Ras isoforms vary in their ability to activate Raf- 1 and phosphoinositide 3-kinase. J Biol Chem 273: 24052-24056, 1998.

28. Tommasi S, Dammann R, Zhang Z, et al: Tumor susceptibility of Rassf1a knockout mice. Cancer Res 65: 92-98, 2005. 